\title{
Ex vivo lung perfusion as a human platform for preclinical small molecule testing
}

\author{
Nathaniel M. Weathington, ${ }^{1}$ Diana Álvarez, ${ }^{1,2}$ John Sembrat, ${ }^{1,2}$ Josiah Radder, ${ }^{1}$ Nayra Cárdenes, ${ }^{1,2}$ \\ Kentaro Noda, ${ }^{3}$ Qiaoke Gong, ${ }^{1}$ Hesper Wong, ${ }^{1}$ Jay Kolls, ${ }^{4}$ Jonathan D'Cunha, ${ }^{3}$ \\ Rama K. Mallampalli, ${ }^{1,5,6}$ Bill B. Chen, ${ }^{1,5}$ and Mauricio Rojas ${ }^{1,2,7}$ \\ 'Department of Medicine, Division of Pulmonary, Allergy, and Critical Care Medicine, ${ }^{2}$ Simmons Center for Interstitial \\ Lung Disease, and 'Department of Cardiothoracic Surgery, University of Pittsburgh, Pittsburgh, Pennsylvania, USA \\ ${ }^{4}$ Department of Medicine, Tulane University, New Orleans, Louisiana. ${ }^{5}$ Department of Cell Biology, University of Pittsburgh, \\ Pittsburgh, Pennsylvania, USA. ${ }^{6}$ The Veterans Affairs Pittsburgh Health System, Pittsburgh, Pennsylvania, USA. \\ ${ }^{7}$ The University of Pittsburgh McGowan Institute for Regenerative Medicine, Pittsburgh, Pennsylvania, USA.
}

\begin{abstract}
The acute respiratory distress syndrome (ARDS) causes an estimated 70,000 US deaths annually. Multiple pharmacologic interventions for ARDS have been tested and failed. An unmet need is a suitable laboratory human model to predictively assess emerging therapeutics on organ function in ARDS. We previously demonstrated that the small molecule BC1215 blocks actions of a proinflammatory $\mathrm{E} 3$ ligase-associated protein, $\mathrm{FBXO3}$, to suppress $\mathrm{NF}-\mathrm{\kappa B}$ signaling in animal models of lung injury. Ex vivo lung perfusion (EVLP) is a clinical technique that maintains lung function for possible transplant after organ donation. We used human lungs unacceptable for transplant to model endotoxemic injury with EVLP for 6 hours. LPS infusion induced inflammatory injury with impaired oxygenation of pulmonary venous circulation. BC1215 treatment after LPS rescued oxygenation and decreased inflammatory cytokines in bronchoalveolar lavage. RNA sequencing transcriptomics from biopsies taken during EVLP revealed robust inflammatory gene induction by LPS with a strong signal for NF-KB-associated transcripts. BC1215 treatment reduced the LPS induction of genes associated with inflammatory and host defense gene responses by Gene Ontology (COterm) and pathways analysis. BC1215 also significantly antagonized LPS-mediated NF$\kappa B$ activity. EVLP may provide a unique human platform for preclinical study of chemical entities such as FBXO3 inhibitors on tissue physiology.
\end{abstract}

Authorship note: NMW, DA, and JS contributed equally to this work. RKM, $\mathrm{BBC}$, and MR contributed equally to this work.

Conflict of interest: RKM and BBC are consultants for Koutif Pharmaceuticals.

Submitted: June 5, 2017

Accepted: August 17, 2018

Published: October 4, 2018

Reference information:

JCI Insight. 2018;3(19): e95515

https://doi.org/10.1172/jci.

insight.95515.

\section{Introduction}

The acute respiratory distress syndrome (ARDS) is a disease process that is often associated with infection, evolves rapidly, and confers a very high mortality on the afflicted $(1,2)$. Advances in clinical care have significantly improved ARDS outcomes in the past 2 decades(3), but new pharmacotherapy for ARDS has not emerged (4). Many investigators believe that ARDS is manifested by an overzealous inflammatory response and that targeted immunosuppression could alleviate the systemic stresses of ARDS and suppress disease progression $(1,5)$. While multiple agents (potentially novel small molecules, corticosteroids, prostaglandins, surfactants, $N$-acetylcysteine, and biologic therapies) have provided promising results in murine and larger-animal lung injury models, human clinical trials have failed to reveal benefit for any of these agents (4). These observations in animals coupled with the excessively high rate of failure of drug candidates underscore a significant need for intact human models that might provide a foundation for prediction of therapeutic efficacy as investigational new compounds track through the preclinical drug pipeline.

Another field in which inflammatory lung injury predicts poor clinical outcomes is lung transplant $(6,7)$ ex vivo lung perfusion (EVLP) is a method of organ preparation that has become prevalent in lung transplant centers around the world in the past 10 years $(8,9)$. Since its inception in $2007(10)$, this methodology has been proven as a means to evaluate and potentially rehabilitate lungs that may have edema or atelectasis and prolong their window for transplant evaluation. EVLP is now used by multiple centers and 
has, overall, been clinically successful to effectively expand the pool of viable organs for transplant (11). The lung transplant community has developed a robust protocol for EVLP that can capture key physiologic parameters (gas exchange, lung mechanics, pulmonary vascular hemodynamics, and edema) (12), and some have expanded EVLP assessment to obtain biospecimens for limited analysis (13). In the current study, we collected pulmonary vascular perfusate, broncho-alveolar lavage fluid (BALF), and tissue biopsies for analysis of tissue, cellular, protein, and genetic markers. As such, this system can provide consistent physiologic readouts at multiple levels of scale from global organ function to gene transcription. Multiple clinical studies have evaluated lungs' performance by EVLP with the intent to transplant, if acceptable physiologic milestones are met. Lee et al. have evaluated isolated perfused lungs experimentally in a model of topical endotoxin injury, with treatment groups given mesenchymal stem cells (MSC) or MSC conditioned media and other modulators (14). Those experiments were performed on inflated lungs without tidal ventilation and demonstrated that MSC preparations improved alveolar fluid clearance, in part, through the action of the epithelial sodium channel (ENaC). EVLP has not, to our knowledge, been used as an experimental model to assess lung function in the setting of injury by conventional parameters (e.g., gas exchange), nor has the effect of any drug been tested using human EVLP as an injury model.

We have recently reported that induction of the ubiquitin E3 ligase protein FBXO3 in inflammation stabilizes the proinflammatory TNF receptor-associated factors (TRAFs) $(15,16)$. TRAFs promote inflammatory signals through activation of the NF- $\mathrm{BB}$ inflammatory transcriptional master regulator. TRAF proteins are constitutively shuttled for proteasomal degradation by the E3 ligase subunit FBXL2. FBXO3 is induced during inflammation to facilitate FBXL2 degradation with a resultant increase in TRAF abundance and signaling. The small molecule FBXO3 inhibitor BC1215 decreases inflammation by blocking the FBXO3 protein to suppress TRAF signaling (Supplemental Figure 1A; supplemental material available online with this article; https://doi.org/10.1172/jci.insight.95515DS1), and BC1215 prevents inflammatory lung injury, paw edema, Pseudomonal lung injury, neuropathic pain, and dextran sulfate sodiuminduced (DSS-induced) colitis in mice (15-17).

In this report, we evaluated EVLP as an experimental platform for endotoxemic lung injury, with instillation of LPS via the pulmonary artery (PA) resulting in a robust cytokine response, along with decreased pulmonary venous oxygen content over 5 hours. Topical administration of BC1215 shortly after LPS exposure preserved pulmonary venous partial pressure of oxygen $\left(\mathrm{pO}_{2}\right)$. Likewise, $\mathrm{BC} 1215$ reduced induction of the Type 1 cytokines IL-1, IL-6, and IL-10 as measured by ELISA of BALF. RNA sequencing (RNA-Seq) analysis revealed that treatment of lungs with BC1215 after LPS exposure significantly blunted the LPS-induced NF- $\mathrm{KB}$ transcriptional response. We conclude that, as observed in cellular and animal studies, BC1215 antagonizes the FBXO3/FBXL2/TRAF inflammatory axis in this human organ model of lung injury.

\section{Results}

BC1215 improves LPS impairment of lung oxygen delivery. We utilized the clinical EVLP platform (XVIVO Systems) as the foundation to our experimental lung injury system with the addition of a PA infusion pump, a bronchoscope, and a biopsy stapler for the collection of specimens over the course of each preparation (Figure 1A). In this system, a perfusion solution was swept through a gas exchange membrane that decreased the $\mathrm{pO}_{2}$ of the PA limb to $\sim 70 \mathrm{mmHg}$. For all experiments, perfusion was started as soon as possible, with lung inflation and recruitment maneuvers performed once tissue had reached $36^{\circ} \mathrm{C}$, followed by the initiation of tidal ventilation (tidal volume of $6 \mathrm{ml} / \mathrm{kg}$ ideal body weight with $5 \mathrm{cmH}_{2} \mathrm{O}$ after expiratory end pressure and a rate of 7 breaths per minute), which we counted as time zero for the EVLP lung injury protocol illustrated in Figure 1B. In LPS experiments, endotoxin was introduced to the pulmonary arterial limb of the EVLP circuit at 1 hour, and $50 \mathrm{ml}$ therapeutic $\mathrm{BC} 1215$ or vehicle was administered topically via a bronchoscope (2-3 $\mathrm{ml}$ per segmental bronchus) 1 hour later. We chose bronchoscopic administration to maximize local tissue concentrations for therapeutic effect, and we chose to avoid direct administration of BC1215 to the perfusate because we did not wish to decrease bioavailability due to albumin or LPS binding of the compound.

Donor demographic data was obtained from our organ procurement agency in a deidentified fashion without significant baseline demographic differences in age or BMI between organ donors (Table 1). Lungs were rejected from transplant consideration for multiple reasons, including suspicion for aspiration or pneumonia (most common), abnormal radiograph (e.g., lung nodules), or developmental abnormality. Physiologic mechanical parameters were recorded throughout the EVLP lung injury protocol for all experiments. PA pressure (PAP) and resistance (Figure 1, C and D) and respiratory mechanics (Figure 1E and Supplemental Figure 1D) revealed 
A

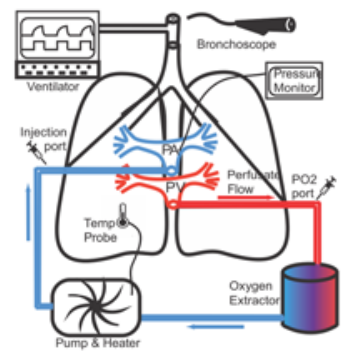

B

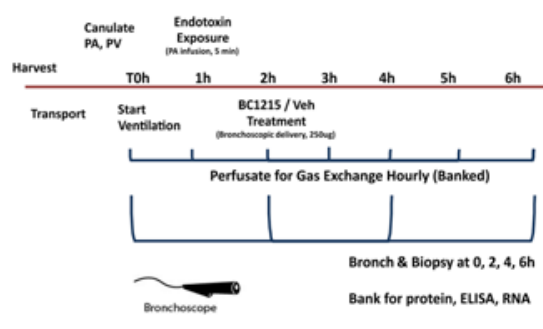

C

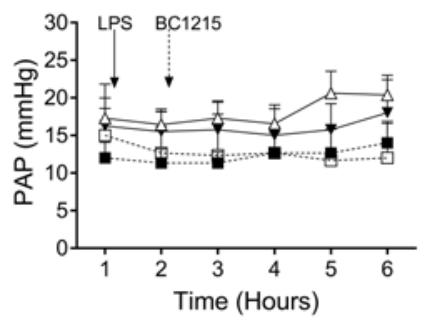

D

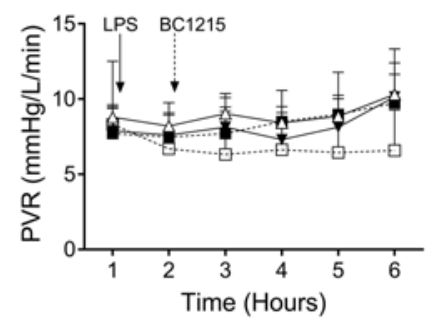

E

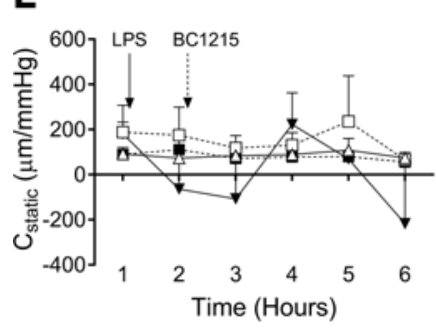

$\mathbf{F}$

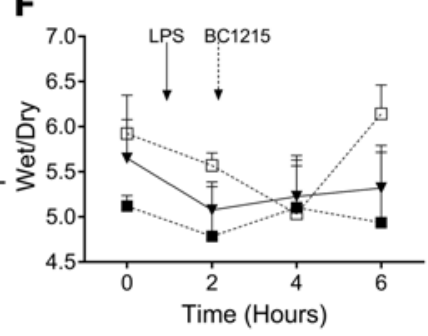

G

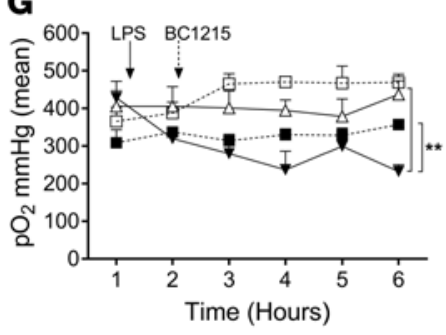

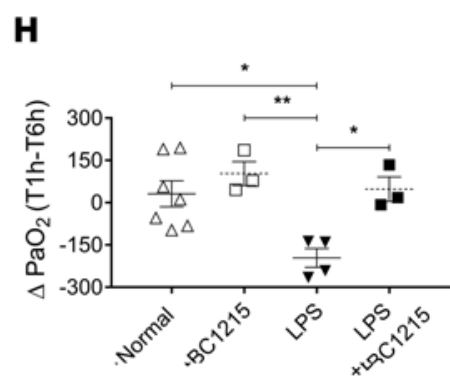

I

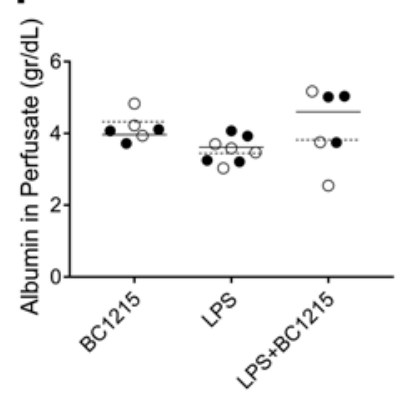

J

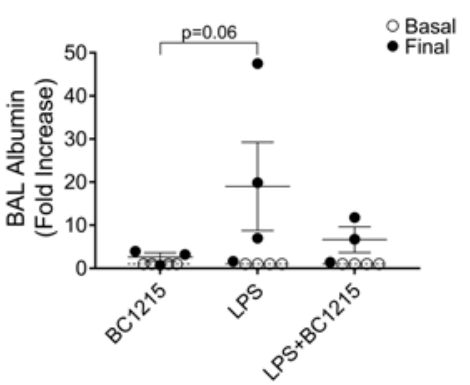

K

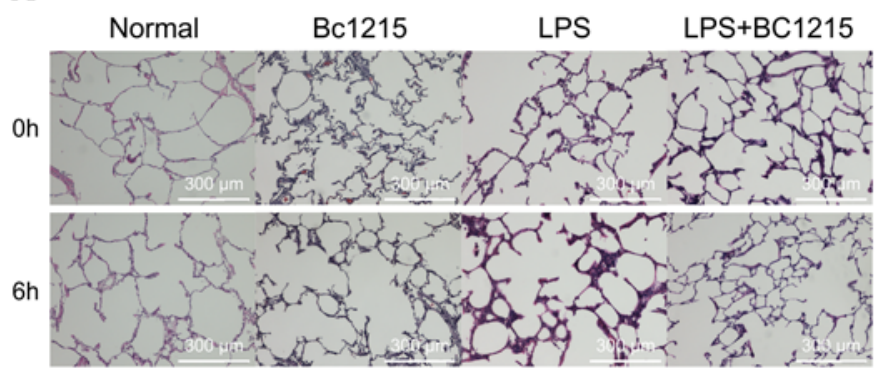

$\star$ Normal $\square$ BC1215 $*$ LPS - LPS $+B C 1215$

Figure 1. LPS induces gas exchange impairment in EVLP that is abrogated by BC1215. (A) The EVLP setup enables delivery of LPS into the pulmonary artery feed with measurement of gas exchange via the pulmonary arterial and venous ports, measurement of ventilator and vascular pressures, and bronchoscopic drug delivery and sampling. (B) Experimental protocols for LPS exposure ( $5 \mu \mathrm{g} / \mathrm{Kg}$ ideal body weight of donor), BC1215 treatment (2.5 mg by bronchoscope), physiology evaluation, and sample collection. (C and $\mathbf{D})$ PAP and resistance were not significantly different between groups over 6 hours of EVLP. (E) Static compliance on EVLP is low across all preparation evaluated. (F) Wet/dry weight ratios of lungs did not differ over time or between groups. (C) Pulmonary vein perfusate oxygen content decreases over time after LPS exposure but remains stable when BC1215 is administered. (H) Change in perfusate oxygenation from 1 hour to 6 hours after ventilation start is significantly decreased by LPS, with normalization by BC1215 treatment (mean \pm SEM; ${ }^{*} P<0.05,{ }^{*} P<0.01$ by 2 -way ANOVA; $n=3$ ). (I) Albumin concentration in perfusate is maintained throughout EVLP. (J) Changes in BAL albumin content increases in the LPS group but not in BC1215-treated preparations. (K) Representative histology micrographs from all conditions.

no significant differences between control lungs (not exposed to LPS), BC1215-treated lungs, LPS-exposed lungs, or LPS-exposed lungs treated with BC1215. Perfusion fluid was evaluated by a blood gas analyzer each hour for $\mathrm{pH}, \mathrm{pO}_{2}$ and $\mathrm{pCO}_{2}$, bicarbonate, and glucose concentrations. While tissue wet/dry ratios were not changed by LPS exposure or BC1215 (Figure 1F), the partial pressure of oxygen in the pulmonary venous limb was significantly depleted over 5 hours after LPS instillation from an average value of $428 \mathrm{mmHg}$ at the time of LPS instillation to an average value of $218 \mathrm{mmHg}$ at the end of the experiment (Figure 1G). This decrease in oxygen content (Figure $1 \mathrm{H})$ was significant compared with normal $(P<0.05)$ or BC1215 $(P<0.01)$ controls, which displayed slight increases in pulmonary venous $\mathrm{O}_{2}$ over the course of EVLP. LPS-exposed BC1215-treated lungs demonstrated unchanged fluid oxygenation over time, without the decreased oxygen transfer we observed in LPS-exposed vehicle-treated preparations $(P<0.05$; Figure 1, G and H, and Supplemental Figure 1, B and $C)$. Because the perfusion solution in these experiments contains physiologic albumin at the start, we tested for increases in albumin concentrations in the alveolar compartment. Albumin concentrations in perfusate fluid did not change over the course of EVLP (Figure 1I), and we observed a trend toward increase in albumin over time in the BAL of lungs exposed to LPS with a $P=0.06$ (Figure 1J), perhaps indicating capillary leak. There was 
A

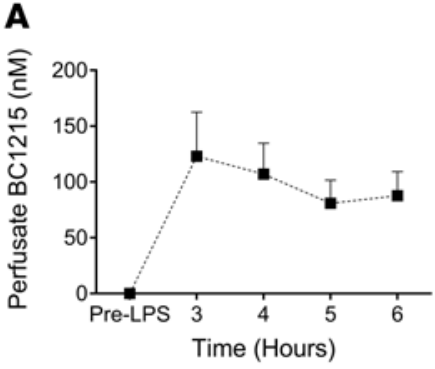

E

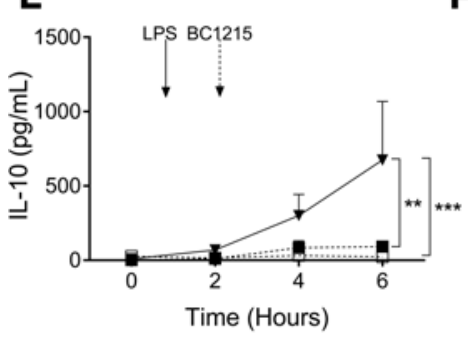

I

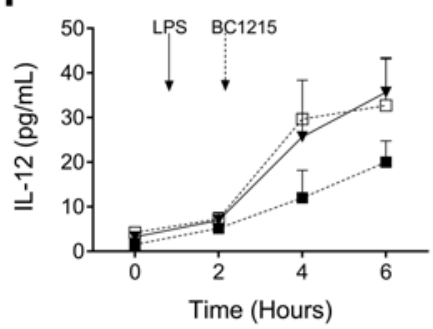

B

$\mathbf{F}$
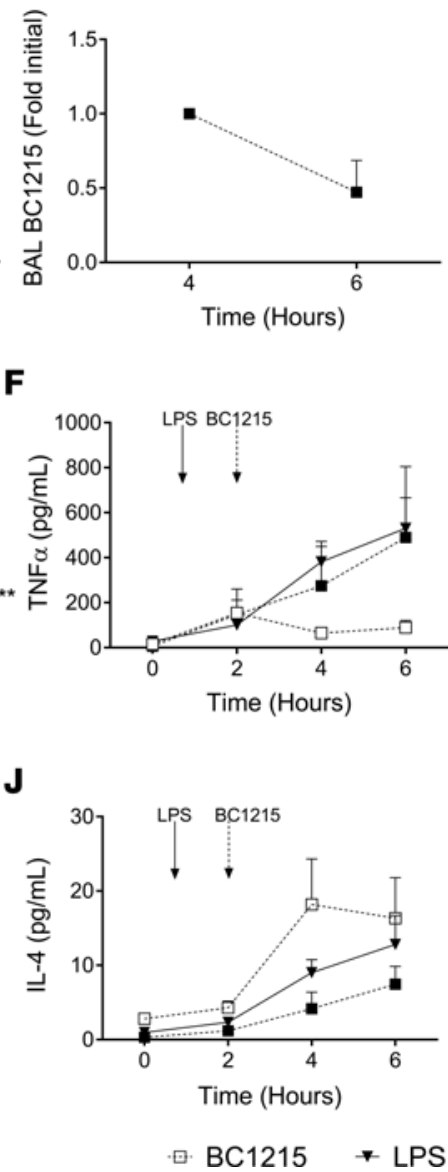

C

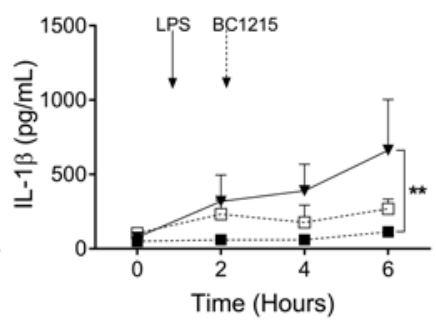

G

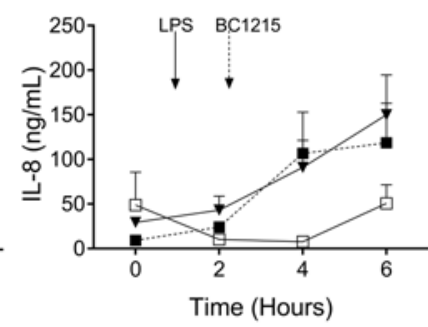

K

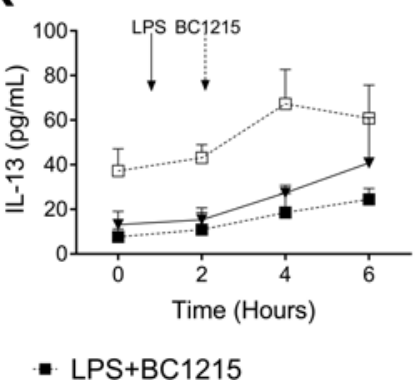

D

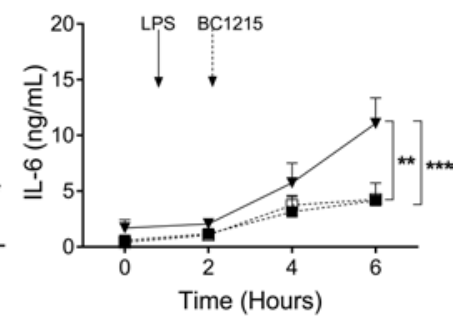

H

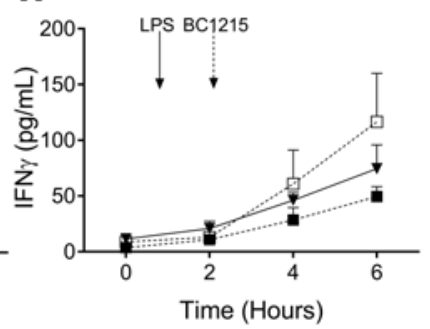

L

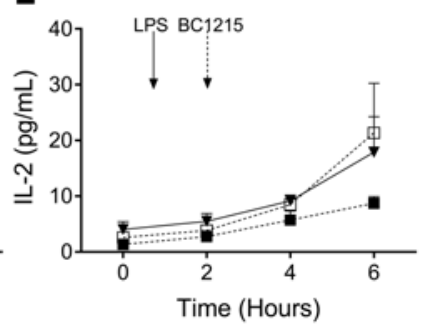

Figure 2. Cytokine induction by LPS is modulated by BC1215 in BALF. (A and B) BC1215 concentration was maintained in the perfusate (A) but decreased in the BALF (B) during EVLP. Cytokines were measured from samples of indicated time points. Type 1 cytokines IL-1 13 , IL-6, and IL-10 show significantly suppressed cytokine levels in BAL (C-E) but not TNF $\alpha$, IFN $\gamma$, or IL-8 (F-H). (I-L) IL-12, IL-13, and IL-4 were also decreased in BC1215-treated preps at 6-hour time point. $n=3$ separate experiments in technical duplicate for each data point shown (mean $\pm \mathrm{SEM}$; 2-way ANOVA ${ }^{* *} P<0.01,{ }^{* *} P<0.001$ ).

no such change in albumin concentrations in the BAL over time in the preparations where only BC1215 was administered. LPS-exposed lungs that were later treated with BC1215 appeared to have less increase in BAL albumin, though our $n$ is too low to gain statistical significance $(P=0.3)$. Lung biopsy specimens were evaluated for edematous changes, which can cause diffusion block and drive impairment in gas exchange, and the histological appearance of EVLP lung tissue shows slightly increased septal thickening at the 6-hour time point in LPS-treated lungs compared with other samples (Figure 1K).

BC1215 rapidly crosses the alveolar space and blunts the inflammatory cytokine response to LPS in EVLP. Bioanalysis of perfusate fluids reveal that BC1215 enters circulation of the EVLP perfusion system to maximal concentrations within 1 hour after bronchoscopic administration, when the average $\mathrm{BC} 1215$ concentration was $110 \mathrm{nM}$. Thereafter, perfusate BC1215 levels dropped an average of $19 \%$ for the 6 experiments, and kinetics are shown in Figure 2A. BAL levels of BC1215 were detectable 2 and 4 hours after administration (at $t=4$ and $t=6$ hours of experiment) and decrease slightly in this interval (Figure 2B). We evaluated cytokine induction after LPS exposure with or without BC1215 treatment from perfusion fluid and BALF specimens collected during the EVLP lung injury protocol. After LPS administration, the majority of cytokines evaluated in our array were increased over time in both the alveolar (Figure 2, C-L) and perfusate (Supplemental Figure 2, A-J) compartments. BC1215 treatment alone did not induce a significant cytokine response, and BC1215 effectively suppressed LPS induction of IL-6, IL-1 $\beta$, and IL-10 in BALF (Figure 2, C-E). Cytokines TNF and IL-8 were not reduced by BC1215 treatment after LPS exposure, and IFN $\gamma$, IL-12, IL-4, IL-13, and IL-2 were minimally induced by EVLP for all conditions. 
BC1215 decreases induction of inflammatory gene transcription after LPS exposure. Because BC1215 was designed as an inhibitor of FBXO3 to destabilize TRAF proteins with consequent suppression of NF- $\mathrm{NB}$ transcriptional activation, we isolated RNA from lung tissue preparations for next-generation sequencing (NGS) and RNA-Seq to compare transcriptional outputs between lung preparations. RNA yield and quality, as well as sequence data quality, was validated per facility protocol. Based on fragments per kilobase of transcript per million (FPKM) values, we averaged the individual samples together into pre-LPS ( $t=0$ hours; $n=6)$, post-LPS $(t=4$ hours, $n=3)$ and post-LPS + BC1215 $(t=4$ hours, $n=3$ ) groups to obtain mean and SD FPKM values for gene expression. We evaluated genes of interest including drug target pathway genes, which revealed trends toward upregulation of proinflammatory genes in the pathway (FBXO3 and TRAFs) with significant induction of TRAF1 by LPS with or without BC1215, without signal change in the FBXL2 transcript for any condition (Figure 3, A-D). Inflammatory cytokines IL-1 $\beta$, IL-6, and IL-8 also displayed transcript upregulation in response to LPS, with a trend toward decreased signal after BC1215 (Figure 3, E-H).

To evaluate the specific transcriptomic changes conferred by BC1215 signal changes, we queried the grouped FPKM data for significant changes in single gene expression between post-LPS vs. post-LPS (+BC1215) conditions, and the top 20 differentially regulated genes between these groups are shown in the heatmap list (Figure 4A). One hundred eighteen genes were significantly upregulated with BC1215, while 110 genes were significantly downregulated compared with the post-LPS group. Bioinformatic screening of the set of BC1215-downregulated transcripts was performed with the DAVID web analysis tool (https://david.ncifcrf.gov/summary.jsp) $(18,19)$. Gene Ontology (GOterm) analysis of those genes downregulated by BC1215 compared with LPS alone revealed significant associations for enrichment of signaling proteins and proteins that contain disulfide bonds (Table 2) with $P$ values approximating the $1 \times 10^{-12}$ range. Such molecules include messenger proteins like cytokines and chemokines. GOterm pathways analysis showed highly significant enrichment with Bonferroni correction of gene products involved with the defense response $\left(P=1 \times 10^{-8}\right)$ and the inflammatory response $(P=1 \times$ $10^{-5}$ ), implying that $\mathrm{BC} 1215$ blunts gene transcription preferentially in these pathways (Table 3 ). This observation was corroborated with STRING pathways analysis of the BC1215-downregulated genes, which populated an association diagram (Figure 4B) based on known functions of the proteins encoded by genes listed. Among the genes downregulated by BC1215, 19\% (21 genes) were previously described

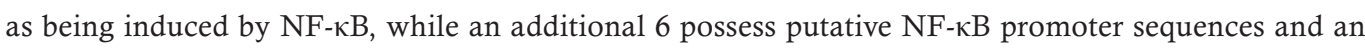
additional 4 genes were characterized as inflammatory proteins by GOterm (Figure 4C).

We hypothesized that, based on TRAF signal suppression, BC1215 would preferentially oppose gene induction induced by LPS through the TRAF/NF-kB axis. We evaluated transcript abundance of the 418 known NF- $\mathrm{BB}$-dependent gene transcripts from an online database (https://www.bu.edu/nf-kb/ gene-resources/target-genes/) among these 3 groups. A heatmap of these data (Figure 5A) revealed normalization of signal intensity in post-LPS preparations treated with BC1215 compared with the post-LPS group without BC1215. LPS induces some genes and represses others among this group, and BC1215 consistently antagonized this effect. Beyond the $21 \mathrm{NF}-\kappa \mathrm{B}$ genes noted above whose LPS induction was significantly suppressed by BC1215, this inhibitor opposed the action of LPS on many

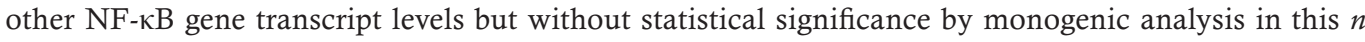
= 3 dataset. Using the FPKM values and SDs from our dataset (Supplemental Table 1), we devised a statistical test to evaluate significance of $\mathrm{BC}-1215$ suppression of the 418 validated $\mathrm{NF}-\kappa \mathrm{B}$ transcription factor targets. This test evaluated the change between FPKM for each gene in the set between pre-LPS and post-LPS conditions and compared that difference with the difference between LPS only and LPS $+\mathrm{BC} 1215$. In this method, the $\triangle$ for each FPKM, its directionality, and SD contributed to a grouped score. In considering the effects of $\mathrm{BC} 1215$ on the set of $\mathrm{NF}-\kappa \mathrm{B}$ genes in aggregate, the suppression of gene induction is significant with a $P$ value of $7.8 \times 10^{-9}$ (Figure $\left.5 \mathrm{~B}\right)$. In order to evaluate the specificity of this observation for NF- $\mathrm{kB}$ genes, we generated a simulation model to examine multiple gene sets of identical size (418 genes) and executed the same statistical test. When we simulated the same statistical test for 100,000 randomly generated gene sets, each containing 418 genes, only 106 of these sets yielded a $P$ value $<0.05$. The NF- $\kappa$ B gene set has a lower $P$ value than any of the gene sets in our simulation by $>3$ orders of magnitude (Figure $5 \mathrm{C}$ ), indicating that the activity of BC1215 specifically silences gene transcriptional changes through the TRAF/NF-kB axis. 

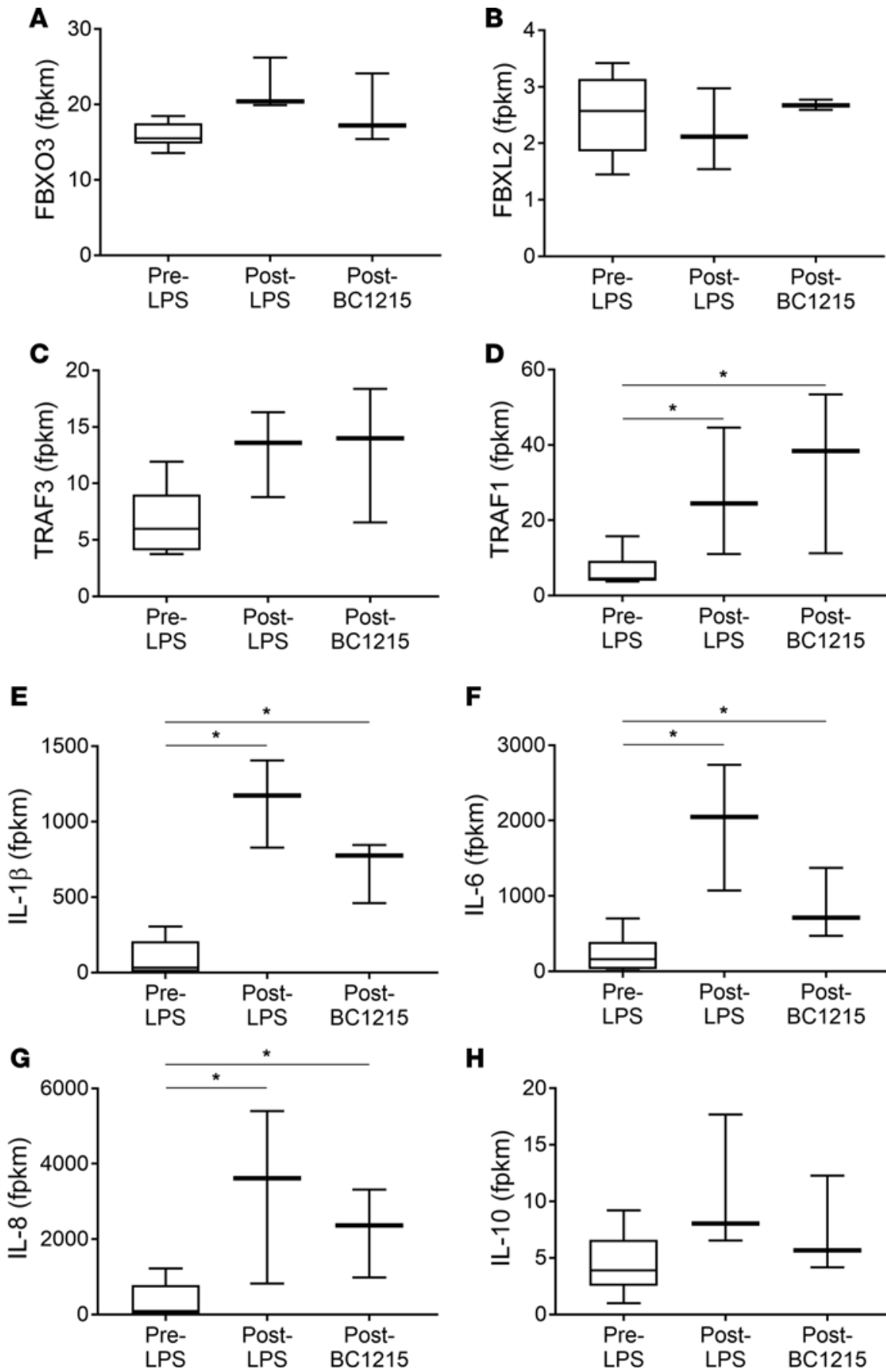

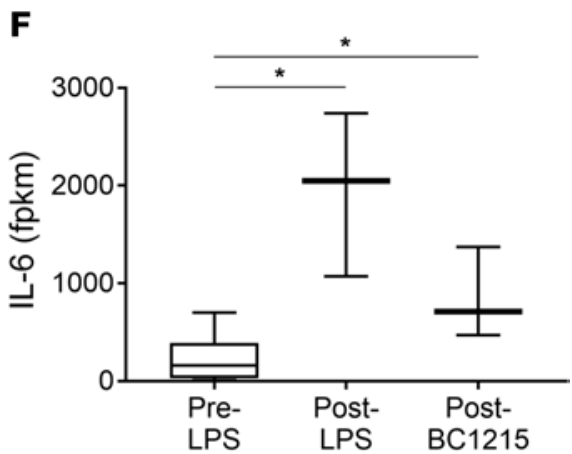

H

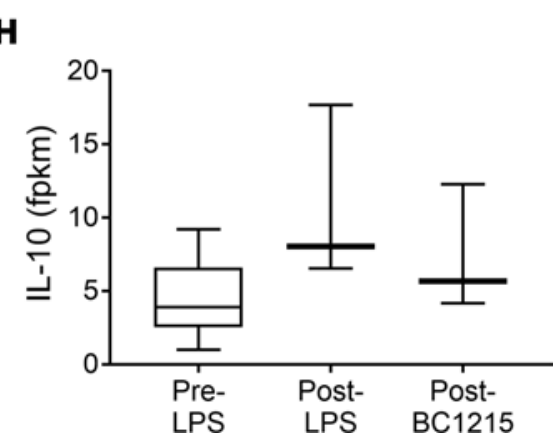

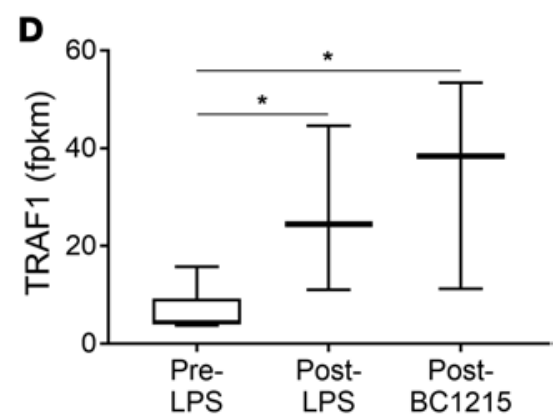

Figure 3. RNA-Seq evaluation of gene transcription after LPS with or without BC1215 treatment. (A-H) Genes of interest analysis show significant induction of several inflammatory genes by LPS with minimal modulation by $\mathrm{BC} 1215$, which targets $\mathrm{FBXO} 3$ activity and protein stability of FBXL2 and TRAFs (box and whisker plots show median FPKM, first and third quartiles, and maximum and minimum values; ${ }^{*} P<0.05$ by 2 -tailed $t$ test as implemented by CuffDiff).

\section{Discussion}

In this study, we have used EVLP to model acute lung injury by LPS exposure and evaluated BC1215 as a suppressor of inflammatory gene and pathway activation. We believe that this study furthers previous work testing perfused lungs to assess preclinical drug candidates.

In the context of other studies using whole human lung preparations, our work displays considerable differences from those previously published, including recent technologies such as lung-on-chip (20). EVLP has been developed by several groups as a means to evaluate donor human lungs with intent to transplant if adequate organ function is achieved in the course of a protocol (12). Early studies reveal that EVLP itself triggers an inflammatory reaction with increases in cytokines after harvest and placement of organs on pump (21). One investigation sought to decrease the amount of inflammatory cytokines present by placing an adsorbent membrane in line within the circuit to trap inflammatory cytokines (22). Our group 
A
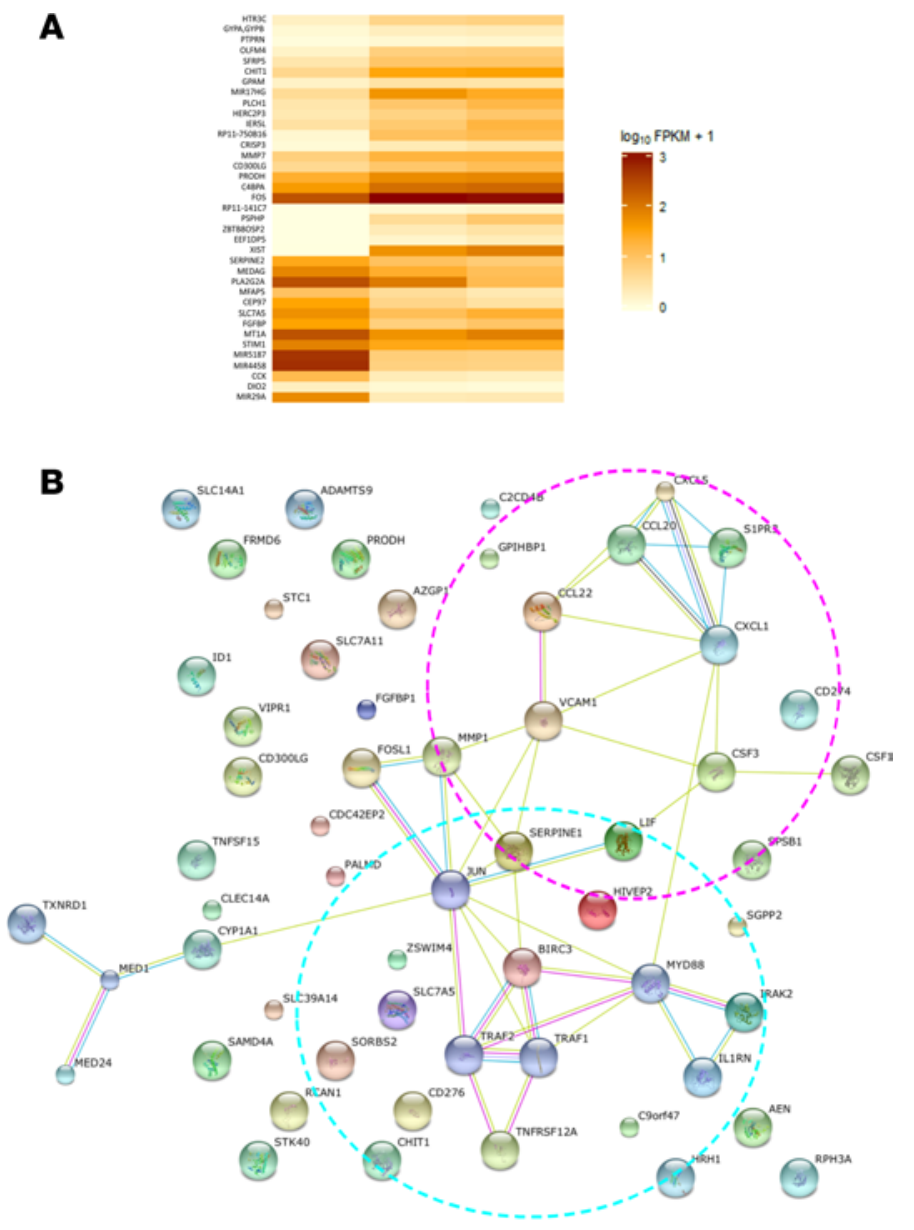

C

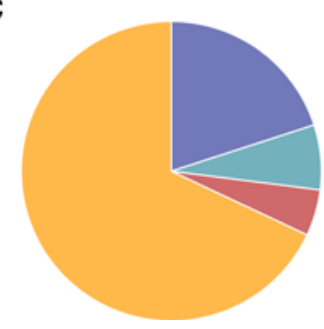

Figure 4. Gene transcription after LPS with or without BC1215 treatment. (A) Heatmap of the top genes changed between post-LPS and post-LPS (+BC1215) conditions ranked by overall significance as determined by two-tailed $t$ testing as implemented by CuffDiff for differential expression testing. (B) STRING analysis of BC1215-suppressed gene products reveals inflammatory cytokine/chemokine (magenta ring) and host defense (cyan ring) signals and implicates TRAF1 and TRAF2 as nodal signal mediators for BC1215 action. (C) The group of mRNA transcripts that were significantly suppressed by BC1215 are over-represented by NF-KBinducible and inflammatory genes.

recently used a leukocyte filter in a rat model to decrease cytokine levels and improve lung function (23), while another group claims such filters offer little benefit in a pig EVLP model (24). Another study delivered high-dose antibiotics to perfused lungs prior to their transplant, with resultant decreases in allograft bacterial burden observed (25). This intervention may prove clinically significant in the practice of clinical EVLP in the coming years. Each of those EVLP studies were conducted without any injury induced, as their endpoints were focused on organ reconditioning and not investigation of organ responses to injury. It is possible that the use of antiinflammatory agents like BC1215 during EVLP might improve organ inflammatory profiles and graft performance in a transplant setting, as we see little induction of inflammatory cytokines in lungs treated with BC1215 alone. Other groups have used animal EVLP models to study lung function and interventions to systemic disease, which are globally informative but do not specifically evaluate human organ responses to therapy, as we have attempted in this study.

Lee et al. have successfully evaluated human lungs in an isolated perfused LPS injury model with evaluation of alveolar fluid clearance (14). Several important differences exist between that study and our own. First, the Lee et al. study added LPS bronchoscopically to the right middle lobe, which would more fittingly act as a surrogate for pneumonia-induced inflammatory injury, while our study exposed lungs to endotoxin via the PA as a model of endotoxemic lung injury in the setting of systemic infection. 
Table 1. Donor demographic data from ex vivo lung perfusion experiments reported

\begin{tabular}{|c|c|c|c|c|c|c|}
\hline & Date & Sex & Age & Height (m) & Weight (Kg) & BMI \\
\hline \multirow[t]{5}{*}{ Normal } & $1 / 17 / 2013$ & Female & 48 & 1.6 & 84 & 32.81 \\
\hline & $10 / 7 / 2013$ & Female & 51 & 1.63 & 71.7 & 26.99 \\
\hline & $12 / 11 / 2013$ & Male & 30 & 1.9 & 93 & 25.76 \\
\hline & $2 / 21 / 2015$ & Male & 40 & 1.7 & 74.84 & 25.90 \\
\hline & $3 / 24 / 2015$ & Male & 37 & 1.85 & 131 & 38.28 \\
\hline & $10 / 09 / 2017$ & Female & 52 & 1.73 & 72 & 24.06 \\
\hline & 10/11/2017 & Male & 30 & 1.8 & 76 & 23.46 \\
\hline \multirow[t]{3}{*}{ LPS } & $4 / 24 / 2015$ & Male & 61 & 1.83 & 91 & 27.17 \\
\hline & $7 / 7 / 2015$ & Male & 25 & 1.85 & 85 & 24.84 \\
\hline & $9 / 27 / 2015$ & Male & 30 & 1.85 & 126.4 & 36.93 \\
\hline
\end{tabular}

Second, the Lee et al. paper maintained lung inflation with static continuous positive airways pressure (CPAP) of $10 \mathrm{cmH}_{2} \mathrm{O}$, while we used the current EVLP clinical protocol of delivering tidal ventilation to the lung preparations with recruitment maneuvers each hour. Third, Lee et al. included $100 \mathrm{ml}$ of whole human blood into their perfusion solution (DMEM media + albumin) to assess leukocyte infiltration during their injury protocol, while our study evaluated intrinsic inflammatory responses from lung parenchyma and resident leukocytes without the addition of human blood products to the perfusion solution (STEEN solution + albumin). Fourth, the endpoints measured between our 2 studies are very different, with Lee et al. evaluating alveolar fluid clearance in the setting of cellular or conditioned media therapy, while we report multiple parameters of lung function, including respiratory mechanics, pulmonary vascular pressures, gas exchange, cytokine induction, and lung tissue transcriptome profiles.

Interestingly, Lee et al. observed an increase in wet/dry tissue weight ratios after local LPS treatment (14), while our whole-organ LPS exposure did not significantly change this metric, despite a decline in oxygenation of perfusion fluid. We did observe a nearly significant increase in albumin transit into the alveolar space after LPS exposure that was absent in BC1215-treated lungs, which could indicate early capillary leak that might block oxygenation. Another hypothesis to explain the difference in $\mathrm{pO}_{2}$ could be that, in addition to diffusion impairment, oxygen consumption by the inflamed organs may be increased.

Table 2. Gene ontology functional classification

\begin{tabular}{lcccc}
\hline Annotation cluster 1 & Enrichment score 11.02 & Count & P value & Benjamini \\
UP_SEQ_FEATURE & Signal peptide & 52 & $1.7 \times 10^{-17}$ & $7.0 \times 10^{-12}$ \\
SP_PIR_KEYWORDS & Signal & 52 & $2.2 \times 10^{-14}$ & $4.7 \times 10^{-12}$ \\
SP_PIR_KEYWORDS & Disulfide bond & 49 & $3.9 \times 10^{-14}$ & $4.2 \times 10^{-12}$ \\
UP_SEQ_FEATURE & Disulfide bond & 47 & $1.9 \times 10^{-13}$ & $3.9 \times 10^{-11}$ \\
GOTERM_CC_FAT & Extracellular region part & 26 & $9.1 \times 10^{-10}$ & $1.2 \times 10^{-7}$ \\
GOTERM_CC_FAT & Extracellular space & 22 & $1.6 \times 10^{-9}$ & $1.0 \times 10^{-7}$ \\
SP_PIR_KEYWORDS & Secreted & 31 & $2.3 \times 10^{-9}$ & $1.6 \times 10^{-7}$ \\
GOTERM_CC_FAT & Extracellular region & 36 & $7.3 \times 10^{-9}$ & $3.1 \times 10^{-7}$
\end{tabular}

Gene ontology functional classification for transcripts significantly decreased in RNA preparations treated with BC1215 after LPS compared with LPS treatement alone ( $P<0.05$ by 2-tailed $t$ test from RNA-Seq data with $n=3$ per group) derived from the DAVID Webtool and Knowledge Base at https://david.ncifcrf.gov/summary.jsp. 
Table 3. Pathway functional annotation

\begin{tabular}{|c|c|c|c|c|}
\hline Annotation cluster 2 & Enrichment score $\mathbf{8 . 1 7}$ & Count & $P$ value & Benjamini \\
\hline GOTERM_BP_FAT & Defense response & 23 & $1.7 \times 10^{-11}$ & $1.5 \times 10^{-8}$ \\
\hline GOTERM_BP_FAT & Inflammatory response & 14 & $1.1 \times 10^{7}$ & $3.1 \times 10^{-5}$ \\
\hline GOTERM_BP_FAT & Response to wounding & 17 & $1.6 \times 10^{-7}$ & $3.4 \times 10^{-5}$ \\
\hline
\end{tabular}

Pathway Functional Annotation based on gene ontology for transcripts significantly decreased in RNA preparations treated with BC1215 after LPS compared with LPS treatment alone ( $P<0.05$ by 2 -tailed $t$ test from RNA-Seq data with $n=3$ per group) derived from the DAVID Webtool and Knowledge Base at https://david.ncifcrf.gov/summary.jsp.

Since our system does not contain erythrocytes (which would function as an oxygen sink), the overall $\mathrm{O}_{2}$ content per $\mathrm{ml}$ of perfusate is much smaller, and moderate $\mathrm{O}_{2}$ consumption would impart larger changes in $\mathrm{pO}_{2}$. For example, using the standard equation for blood oxygen content, $\mathrm{CaO}_{2}=\left(\mathrm{SaO}_{2} \times\right.$ hemoglobin $\times 1.34)+.003\left(\mathrm{pO}_{2}\right)$, where $\mathrm{SaO}_{2}$ indicates oxygen saturation, the oxygen content of $1 \mathrm{~g}$ of perfusion solution with a $\mathrm{pO}_{2}$ of $333 \mathrm{mmHg}$ is only $1 \mathrm{ml}$, whereas that of fully oxygenated blood with hemoglobin of 10 and $\mathrm{pO}_{2}=333$ is $14.4 \mathrm{ml}$. Consumption of $0.3 \mathrm{ml} \mathrm{O}_{2}$ per gram of fluid would drop the oxygen content by $100 \mathrm{mmHg}$ in fluid with minimal effect if red cells were present. This hypothesis implicates a state that some refer to as cytopathic dysoxia, which results from deranged mitochondrial physiology in sepsis and injury models $(26,27)$. Future studies with this model could evaluate mitochondrial function and energetics during EVLP as a determinant of injury and therapeutic benefit.

The activity of BC1215 in this system suppressed cytokine signaling and limited tissue gas exchange impairment. This finding is consistent with the animal models, in which we have previously observed BC1215 anti-inflammatory activity $(15,16)$. While our previous studies have evaluated whole animal treatment responses or human PBMC, this study enables monitoring of organ physiology and tissue response to inflammatory injury in a human system. BC1215 suppression of the cytokine responses to LPS is consistent with prior studies, and the evaluation of transcript abundance through NGS corroborates the mechanism of BC1215 activity as a suppressor of NF- $\mathrm{KB}$ activation via the FBXO3/FBXL2/TRAF pathway.

While our findings are encouraging for the use of EVLP to investigate new interventions in lung injury preclinically, some significant limitations in our study must be noted. As noted above, this preparation relies only on inflammatory injury intrinsic to the lungs studied, without evaluating the effects of circulating leukocytes. In ARDS, much of the injury from systemic infection is thought to be mediated through the action of infiltrating neutrophils and other cells. However, the tissue response to endotoxin drives some infiltration of circulating cells, blood leukocyte responses may vary greatly between individuals, and ARDS does occur in neutropenic patients $(28,29)$. The genotypic and phenotypic variability between individual donors and organ preparations can be significant, and this study might be strengthened if experimental personnel were blinded to the experimental conditions. While our findings indicate efficacy of BC1215 in countering the LPS-induced NF- $\mathrm{BB}$ activation, conclusions drawn from this small preclinical study are by no means definitive in predicting $\mathrm{FBXO} 3$ inhibition as a therapeutic for humans.

In conclusion, our study indicates that $\mathrm{BC} 1215$ is effective in blunting the lung tissue-specific response to LPS and might reduce injury in the setting of systemic infectious toxicity. Future studies will entail testing FBXO3 inhibition as therapy for inflammatory injury in other preclinical models, and E3 ligase inhibitor therapy may soon enter early first-in-human studies.

\section{Methods}

EVLP. This technique has been described elsewhere in detail (3-5). Briefly, following informed consent from donors or their families, lungs were procured under an established protocol from the University of Pittsburgh Committee for Oversight of Research and Clinical Training Involving Decedents (CORID). Most of these organs were considered for transplant but refused by the surgical team due to abnormal radiographic findings including consolidation or putative pneumonia, lung nodules, or lymphadenopathy that could be neoplastic. For these experiments, donor lungs were assigned to either LPS or LPS + BC1215 protocols in alternating fashion for 7 consecutive studies. BC1215-only experiments were later performed as 3 consecutive experiments. Lungs from patients without preexisting lung disease were procured according to standard 
A

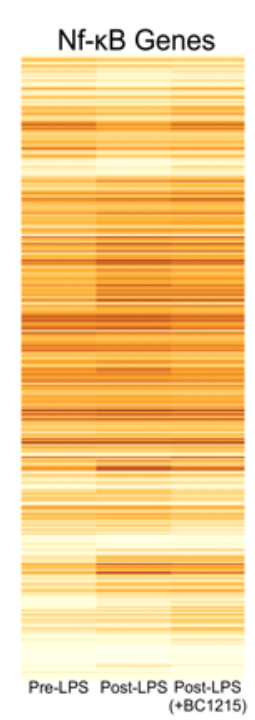

B

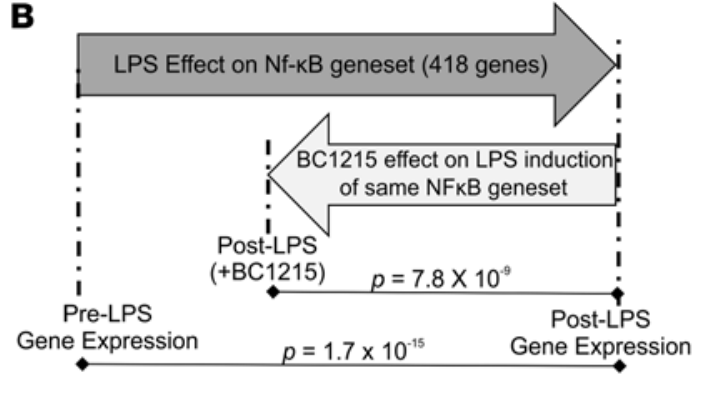

C

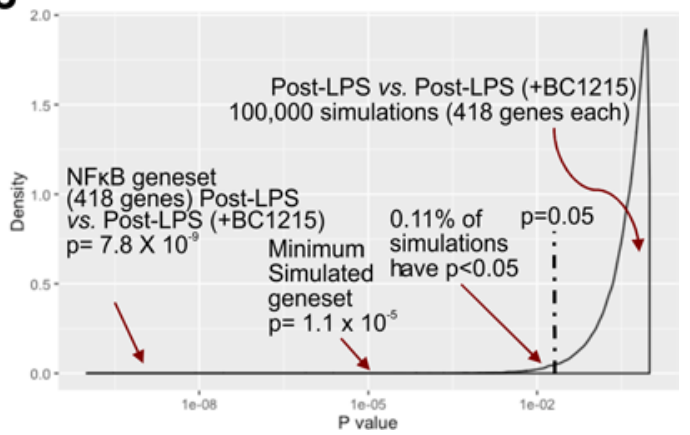

Figure 5. Analysis of NF- $\kappa$ B transcriptional modulation by LPS and antagonism by BC1215. (A) Heatmap showing group averaged fold induction FPKM for each of the 418 described NF-KB-inducible genes. (B) Effect calculations with significance for the NF- $\kappa B$ gene set induction between conditions shown. (C) $P$ value distribution for simulated gene set comparisons between post-LPS and post-LPS (+BC1215) among 100,000 randomly generated transcript sets each containing 418 genes. The NF-אB gene set $P$ value is $1 \times 10^{3}$ below the lowest simulated gene set comparison's significance (2-tailed $t$ test).

clinical protocols and flushed with Perfadex (XVIVO Perfusion AB) using anterograde and retrograde flow and were then placed on ice and transferred to the perfusion center. The ischemia time was between 4-8 hours in both groups. Half an hour before initiating lung perfusion, the perfusion system was set up using a Cobe 43600-000 cardiac bypass pump and Biomedicus 540 pump. The lungs were placed on a draining surface (XVIVO perfusion), and the cannula lines and Silicone Y tubing (XVIVO perfusion) were anastomosed surgically to the left atrium (LA) and PA in sterile fashion. The pulmonary circulation was purged of air, and anterograde and retrograde flush was performed. Perfusion was then started at $10 \%$ of the calculated goal flow (total goal flow $=40 \%$ of donor cardiac output), and continuous pressure flow was increased to goal over an hour. Two liters of perfusion solution (electrolytes matching human plasma $+250 \mathrm{ml}$ of clinical grade $25 \%$ human albumin and $300 \mathrm{mg} / \mathrm{dl}$ glucose), containing 10,000 units heparin was added to prime the circuit, and a Hemotherm 400MR (CSZ Medical) heater/cooler was connected to the oxygenator, with warming increased at $1^{\circ} \mathrm{C}$ every 2 minutes after perfusion start to $38^{\circ} \mathrm{C}$. PAP, pulmonary venous pressure (PVP), and temperature were monitored continuously with a transducer and thermometer probe connected to an HP Viridia 24CT clinical monitor. A detailed logbook was maintained for each preparation to tabulate all recorded values. Only lungs that met inclusion criteria for the protocol $\left(\mathrm{pO}_{2}>250 \mathrm{mmHg},<20\right.$ pack years tobacco history, $<6$ hours cold ischemic time, absent from previously diagnosed lung disease, and not suitable for clinical use) were used. Flow measurements were notated hourly from the pump display into the experimental log. Ventilation was initiated when organ temperature reached $32^{\circ} \mathrm{C}$ (usually 20-30 minutes after perfusion start) with a Puritan Bennett 840 ventilator set to: volume control ventilation with tidal volume of $6 \mathrm{ml} / \mathrm{kg}$ (donor's ideal body weight), 10 breaths per minute, and a positive end-expiratory pressure (PEEP) of $5 \mathrm{~cm} \mathrm{H}_{2} \mathrm{O}$ and $21 \% \mathrm{FiO}_{2}$. The lungs were loosely wrapped in plastic once they were inflated sufficiently to return $80 \%$ of inspired tidal volume. Each hour, maximal gas exchange was evaluated with an increase in $\mathrm{FiO}_{2}$ to $100 \%$ and tidal volume (Vt) to $10 \mathrm{ml} / \mathrm{kg}$ ideal weight for 10 minutes, with mechanical parameters logged. Pulmonary venous gas measurement was recorded using a ABL80 FLEX with Co-oXimetry (Radiometer) that was calibrated daily. BAL (from right middle lobe [RML] and lingula) and tissue biopsy samples (from bilateral lower lobes) were collected at $0,2,4$, and 6 (hours) with clinical disposable bronchoscopes (Ambu) and an Endo GIA autosuture surgical stapler (Tyco) without significant air leak. Biopsy samples were flash frozen and fixed for histologic analysis or placed in RNAlater for RNA extraction 
and analysis. For LPS injury experiments, E. coli 055:B5 endotoxin (MilliporeSigma; all experiments from same container) was diluted in $20 \mathrm{ml}$ sterile PBS at $5 \mu \mathrm{g} / \mathrm{kg}$ ideal body weight for the donor and infused into the PA limb of the circuit over 5 minutes via automated syringe pump at $t=1$ hour. BC1215 was obtained from Dr. Chen's laboratory and is $95 \%$ pure by thin layer chromatography analysis and as stored at $5 \mathrm{mg} /$ $\mathrm{ml}$ in PBS with 1\% DMSO. For BC1215 delivery, $2.5 \mathrm{mg}$ of BC1215 was dissolved in $50 \mathrm{ml}$ sterile PBS, and BC1215 or PBS alone was administered via a bronchoscope with 2-3 $\mathrm{ml}$ of solution directed into each segmental bronchus immediately following $t=2$ hours BAL and biopsy collection.

Measurement of biomarkers. The concentrations of cytokines in the BAL and perfusion solution were measured using multiplexed plate-based immunoassays (MesoScale Discovery) at the University of Pittsburgh Cancer Institute (UPCI) Cancer Biomarkers Facility Core Laboratory. The collected samples were centrifuged at 7,000 g, and supernatant was collected. Samples were analyzed according to the manufacturer's instructions (MesoScale Discovery). Every sample was run in duplicate, and all concentrations were expressed in pg/ml. Albumin concentrations were measured using a Bromocresol Green colorimetric assay (MilliporeSigma). BC1215 concentrations were measured by liquid chromatography-tandem mass spectrometry (LC-MS/MS) Bioanalysis at Touchstone Biosciences.

Histology. Biopsy specimens were placed in $2 \%$ paraformaldehyde and processed by the University of Pittsburgh pathology core and processed by standard techniques. Micrographs were obtained using a Nikon Eclipse microscope with CCD camera.

$R N A$-Seq. Tissues were homogenized and RNA collected via Qiazol, and total RNA was extracted with the RNeasy kit (Qiagen) according to manufacturer's instructions. Samples were sequenced on an Illumina HiSeq 3000 NGS platform by the Health Sciences Sequencing Core at Children's Hospital of Pittsburgh. Reads were preprocessed with Trimmomatic (Version 0.36), and quality was assessed using FASTQC (Version 1.1) (30). Alignment, splice junction mapping, and expression analysis was done using TopHat (Version 2.1.1) and Cufflinks (Version 2.2.1) with GRCH37 as a reference genome (31). CummeRbund (Version 2.12.1) was used for visualization of these results. RNA-Seq data are archived in the NCBI Gene Expression Omnibus (GEO), accession no. GSE118566).

Statistics. For data in Figures 1, 2, and 4, statistical tests described in legends were performed using Graphpad Prism, and for RNA-Seq data, R (Version 3.2.4) was used for additional statistical analysis. Statistics for measured biomarkers were performed with Graphpad Prism using ANOVA for comparisons between multiple groups. Statistics from measured biomarkers were performed using 2-way ANOVA for comparisons between multiple groups and 2-tailed $t$ tests when comparing 2 groups. For RNA-Seq data, all $P$ values were generated using CuffDiff, which utilizes a 2-tailed $t$ test.

Study approval. The use of human tissue in this study is IRB exempt, and institutional approval was granted by protocol 736 of the University of Pittsburgh CORID.

\section{Author contributions}

NW, DA, JS, NC, KN, MR, and JD designed and performed EVLP experiments. JD, JS, KN, and NS prepared organs surgically for EVLP. NW, JS, QG, NC, DA, JK, HW, and WH processed and analyzed samples. NW, DA, JS, JR, BC, RM, and MR analyzed and presented data. NW wrote the manuscript with editorial assistance from RM, MR, BC, and JD. NW, MR, NS, JD, JK, BC, and RM provided equipment and funding. $\mathrm{BC}$ and $\mathrm{RM}$ provided $\mathrm{BC} 1215$.

\section{Acknowledgments}

The authors would like to acknowledge the western Pennsylvania Center for Organ Research and Education (CORE), The University of Pittsburgh Medical Center departments of Respiratory Therapy and Perfusion, and organ donors around the world. K08HL126135 (NW), American Lung Association Biomedical Research Grant (NW), R35 HL139860 (BBC), R01 HL133184 (BBC), and R01 HL123766 (MR) supported this research. Special thanks to the cardiothoracic surgical fellows involved in procurement of the lungs: Sandeep Sainathan, Balikrishnan Mahesh, and Tanuntorn Songdechakraiwut.

Address correspondence to: Nathaniel M. Weathington, Department of Medicine, University of Pittsburgh, 3459 Fifth Avenue, NW 628MUH, Pittsburgh, Pennsylvania 15213, USA. Phone: 412.624.8669; Email: weathingtonnm@upmc.edu. 
1. Piantadosi CA, Schwartz DA. The acute respiratory distress syndrome. Ann Intern Med. 2004;141(6):460-470.

2. Rubenfeld GD, et al. Incidence and outcomes of acute lung injury. N Engl J Med. 2005;353(16):1685-1693.

3. Acute Respiratory Distress Syndrome Network, et al. Ventilation with lower tidal volumes as compared with traditional tidal volumes for acute lung injury and the acute respiratory distress syndrome. N Engl J Med. 2000;342(18):1301-1308.

4. Tonelli AR, Zein J, Adams J, Ioannidis JP. Effects of interventions on survival in acute respiratory distress syndrome: an umbrella review of 159 published randomized trials and 29 meta-analyses. Intensive Care Med. 2014;40(6):769-787.

5. Suter PM. Lung Inflammation in ARDS--friend or foe? N Engl J Med. 2006;354(16):1739-1742.

6. Fisher AJ, et al. Elevated levels of interleukin-8 in donor lungs is associated with early graft failure after lung transplantation. Am J Respir Crit Care Med. 2001;163(1):259-265.

7. Kaneda $\mathrm{H}$, et al. Pre-implantation multiple cytokine mRNA expression analysis of donor lung grafts predicts survival after lung transplantation in humans. Am J Transplant. 2006;6(3):544-551.

8. Van Raemdonck D, Neyrinck A, Cypel M, Keshavjee S. Ex-vivo lung perfusion. Transpl Int. 2015;28(6):643-656.

9. Cypel M, et al. Normothermic ex vivo lung perfusion in clinical lung transplantation. N Engl J Med. 2011;364(15):1431-1440.

10. Steen S, et al. First human transplantation of a nonacceptable donor lung after reconditioning ex vivo. Ann Thorac Surg. 2007;83(6):2191-2194.

11. Valenza F, et al. Ex vivo lung perfusion to improve donor lung function and increase the number of organs available for transplantation. Transpl Int. 2014;27(6):553-561

12. Sanchez PG, Rajagopal K, Pham SM, Griffith BP. Defining quality during ex vivo lung perfusion: The University of Maryland experience. J Thorac Cardiovasc Surg. 2015;150(5):1376-1377.

13. Andreasson AS, et al. Profiling inflammation and tissue injury markers in perfusate and bronchoalveolar lavage fluid during human ex vivo lung perfusion. Eur J Cardiothorac Surg. 2017;51(3):577-586.

14. Lee JW, Fang X, Gupta N, Serikov V, Matthay MA. Allogeneic human mesenchymal stem cells for treatment of E. coli endotoxin-induced acute lung injury in the ex vivo perfused human lung. Proc Natl Acad Sci USA. 2009;106(38):16357-16362.

15. Chen BB, et al. A combinatorial $\mathrm{F}$ box protein directed pathway controls TRAF adaptor stability to regulate inflammation. Nat Immunol. 2013;14(5):470-479.

16. Mallampalli RK, et al. Targeting F box protein Fbxo3 to control cytokine-driven inflammation. J Immunol. 2013;191(10):5247-5255.

17. Lin TB, et al. Fbxo3-Dependent Fbx12 Ubiquitination Mediates Neuropathic Allodynia through the TRAF2/TNIK/GluR1 Cascade. J Neurosci. 2015;35(50):16545-16560.

18. Huang da W, Sherman BT, Lempicki RA. Systematic and integrative analysis of large gene lists using DAVID bioinformatics resources. Nat Protoc. 2009;4(1):44-57.

19. Huang da W, Sherman BT, Lempicki RA. Bioinformatics enrichment tools: paths toward the comprehensive functional analysis of large gene lists. Nucleic Acids Res. 2009;37(1):1-13.

20. Menshykau D. Emerging technologies for prediction of drug candidate efficacy in the preclinical pipeline. Drug Discov Today. 2017;22(11):1598-1603.

21. Sadaria MR, et al. Cytokine expression profile in human lungs undergoing normothermic ex-vivo lung perfusion. Ann Thorac Surg. 2011;92(2):478-484

22. Kakishita T, et al. Suppression of inflammatory cytokines during ex vivo lung perfusion with an adsorbent membrane. Ann Thorac Surg. 2010;89(6):1773-1779.

23. Noda K, et al. Targeting Circulating Leukocytes and Pyroptosis During Ex Vivo Lung Perfusion Improves Lung Preservation. Transplantation. 2017;101(12):2841-2849.

24. Luc JGY, et al. A Leukocyte Filter Does Not Provide Further Benefit During Ex Vivo Lung Perfusion. ASAIO J. 2017;63(5):672-678

25. Nakajima D, et al. Ex Vivo Perfusion Treatment of Infection in Human Donor Lungs. Am J Transplant. 2016;16(4):1229-1237.

26. Singer M. Metabolic failure. Crit Care Med. 2005;33(12 Supp1):S539-S542.

27. Chen BB, et al. E3 ligase subunit Fbxo15 and PINK1 kinase regulate cardiolipin synthase 1 stability and mitochondrial function in pneumonia. Cell Rep. 2014;7(2):476-487.

28. Ognibene FP, et al. Adult respiratory distress syndrome in patients with severe neutropenia. N Engl J Med. 1986;315(9):547-551.

29. Maunder RJ, Hackman RC, Riff E, Albert RK, Springmeyer SC. Occurrence of the adult respiratory distress syndrome in neutropenic patients. Am Rev Respir Dis. 1986;133(2):313-316.

30. Bolger AM, Lohse M, Usadel B. Trimmomatic: a flexible trimmer for Illumina sequence data. Bioinformatics. 2014;30(15):2114-2120.

31. Trapnell C, et al. Differential gene and transcript expression analysis of RNA-seq experiments with TopHat and Cufflinks. Nat Protoc. 2012;7(3):562-578. 